\title{
The Last Theorem of Pierre de Fermat. A short and Simple Proof.
}

Of course Mr. Pierre de Fermat did not know the ABC hypothesis, let alone hypothesis Taniyama-Shimura. He simply wrote that there is no solution in integers $x^{n}+y^{n}=z^{n}$ if $\mathrm{n} \succ 2$ He understood the essence of the proof, but did not even begin to uncover it, assuming it is elementary.

Perhaps like this:

\section{Option 1}

$$
x^{n}+y^{n}=z^{n} \quad(x, y, z, n \in N)
$$

a) $\sqrt{x^{n}}=\sqrt{z^{n}-y^{n}}$ if $n \succ 2 \Rightarrow \sqrt{x^{n}}=x \sqrt{x^{n-2}}$

$$
\begin{gathered}
\sqrt{y^{n}}=\sqrt{z^{n}-x^{n}} \text { if } n \succ 2 \Rightarrow \sqrt{y^{n}}=y \sqrt{y^{n-2}} \\
\sqrt{z^{n}}=\sqrt{y^{n}+x^{n}} \text { if } n \succ 2 \Rightarrow \sqrt{z^{n}}=z \sqrt{z^{n-2}}
\end{gathered}
$$

b) $\frac{x \sqrt{x^{n-2}}}{y \sqrt{y^{n-2}}}=\frac{\sqrt{z^{n}-y^{n}}}{\sqrt{z^{n}-x^{n}}} \Rightarrow \frac{x}{y}=\frac{\sqrt{y^{n-2}} \sqrt{z^{n}-y^{n}}}{\sqrt{x^{n-2}} \sqrt{z^{n}-x^{n}}}$

$$
\frac{z \sqrt{z^{n-2}}}{y \sqrt{y^{n-2}}}=\frac{\sqrt{x^{n}+y^{n}}}{\sqrt{z^{n}-x^{n}}} \Rightarrow \frac{z}{y}=\frac{\sqrt{y^{n-2}} \sqrt{x^{n}+y^{n}}}{\sqrt{z^{n-2}} \sqrt{z^{n}-x^{n}}}
$$

c) $\mathrm{kx}=\sqrt{y^{n-2}} \sqrt{z^{n}-y^{n}} \Rightarrow \mathrm{k}=\frac{\sqrt{y^{n-2}} \sqrt{z^{n}-y^{n}}}{x}$

$$
\mathrm{ky}=\sqrt{x^{n-2}} \sqrt{z^{n}-x^{n}} \Rightarrow \mathrm{k}=\frac{\sqrt{x^{n-2}} \sqrt{z^{n}-x^{n}}}{y}=\frac{\sqrt{y^{n-2}} \sqrt{z^{n}-y^{n}}}{x}
$$

$k \neq K$

$\Rightarrow \boldsymbol{K y}=\mathrm{x} \Rightarrow K=\frac{x}{y}, \operatorname{but}(x, y)=1 \Rightarrow K \notin N \Rightarrow K=1 \Rightarrow x=y$, but $x \neq y$

$$
\begin{aligned}
& \boldsymbol{K}=1 \Rightarrow \sqrt{y^{n-2}} \sqrt{z^{n}-y^{n}}=\sqrt{x^{n-2}} \sqrt{z^{n}-x^{n}} \Rightarrow y^{n-2} x^{n}=x^{n-2} y^{n} \Rightarrow \frac{y^{n} x^{n}}{y^{2}}=\frac{x^{n} y^{n}}{x^{2}} \\
& \Rightarrow x=y \text { but } x \neq y
\end{aligned}
$$

Q.E.D.

\section{Option 2}

$x^{n}+y^{n}=z^{n}$

a) $\sqrt{x^{n}}=\sqrt{z^{n}-y^{n}}$ if $n \succ 2 \Rightarrow \sqrt{x^{n}}=x \sqrt{x^{n-2}}$

$$
\sqrt{y^{n}}=\sqrt{z^{n}-x^{n}} \text { if } n \succ 2 \Rightarrow \sqrt{y^{n}}=y \sqrt{y^{n-2}}
$$




$$
\sqrt{z^{n}}=\sqrt{x^{n}+y^{n}} \text { if } n \succ 2 \Rightarrow \sqrt{z^{n}}=z \sqrt{z^{n-2}}
$$

b) $\frac{x \sqrt{x^{n-2}}}{y \sqrt{y^{n-2}}}=\frac{\sqrt{z^{n}-y^{n}}}{\sqrt{z^{n}-x^{n}}} \Rightarrow \frac{x}{y}=\frac{\sqrt{y^{n-2}} \sqrt{z^{n}-y^{n}}}{\sqrt{x^{n-2}} \sqrt{z^{n}-x^{n}}}$

c) $\frac{x \sqrt{x^{n-2}}}{z \sqrt{z^{n-2}}}=\frac{\sqrt{z^{n}-y^{n}}}{\sqrt{y^{n}+x^{n}}} \Rightarrow \frac{x}{z}=\frac{\sqrt{z^{n-2}} \sqrt{z^{n}-y^{n}}}{\sqrt{x^{n-2}} \sqrt{y^{n}+x^{n}}}$

d) $\left(\sqrt{z^{n}}\right)^{2}=\left(\sqrt{x^{n}}\right)^{2}+\left(\sqrt{y^{n}}\right)^{2} \Rightarrow \frac{\sqrt{x^{n}}}{\sqrt{z^{n}}}=\cos \beta$

$$
\frac{x}{z}=\frac{\sqrt{z^{n-2}} \sqrt{z^{n}-y^{n}}}{\sqrt{x^{n-2}} \sqrt{y^{n}+x^{n}}}=\frac{\sqrt{z^{n-2}}}{\sqrt{x^{n-2}}} \cos \beta \Rightarrow
$$

$k z=\sqrt{x^{n-2}}, \Rightarrow k=\frac{\sqrt{x^{n-2}}}{z},(x, z)=1 \Rightarrow k \notin N \Rightarrow z=\sqrt{x^{n-2}}, \operatorname{but}(x, z)=1 \Rightarrow$ Q.E.D.

and :

$k x=\sqrt{z^{n-2}} \cos \beta \Rightarrow k=\frac{\sqrt{z^{n-2}} \sqrt{x^{n}}}{x \sqrt{z^{n}}} \Rightarrow \frac{\sqrt{x^{x-2}}}{z}=\frac{\sqrt{z^{n-2}} \sqrt{x^{n}}}{x \sqrt{z^{n}}}, \sqrt{x^{n-2}} \prec \sqrt{z^{n-2}} \sqrt{x^{n}} \Rightarrow$ $k \neq K$

$\sqrt{z^{n-2}} \sqrt{x^{n}}=K \sqrt{x^{n-2}} \Rightarrow K=\frac{\sqrt{z^{n-2}} \sqrt{x^{n}}}{\sqrt{x^{n-2}}}=\frac{\sqrt{z^{n-2}}}{x} \notin N \Rightarrow K=1$

but

$(x, z)=1 \Rightarrow$ Q.E.D.

and:

$x \sqrt{z^{n}}=K z \Rightarrow K=\frac{x \sqrt{z^{n}}}{z} \Rightarrow \frac{x \sqrt{z^{n}}}{z}=\frac{\sqrt{z^{n-2}}}{x} \Rightarrow x^{2} \sqrt{z^{n-2}}=\sqrt{z^{n-2}} \Rightarrow x^{2}=1$

but

$x \succ 1 \Rightarrow K=1$

AND:

If $K=1 \Rightarrow \sqrt{z^{n-2}} \sqrt{x^{n}}=\sqrt{x^{n-2}} \Rightarrow \frac{\sqrt{z^{n}}}{z}=\frac{\sqrt{x^{n-2}}}{\sqrt{x^{n}}} \prec 1 \Rightarrow \frac{\sqrt{z^{n}}}{z} \prec 1$,

But $n \succ 2$

Q.E.D.

I am convinced that there can be still be many ways found how to define the relationship between $\mathrm{x}, \mathrm{y}$ and $\mathrm{z}$, proving that Mr. Fermat is correct. 
Also Andrew Beal's hypothesis cannot be forgotten, his genius statement cannot be left without awe. How he managed to do it I cannot comprehend - beautifully and with elegance! This proof confirms that Andrew Beal's hypothesis is true.

Edvins Kozlovskis

Jurmala, LATVIA

30.10 .2016 